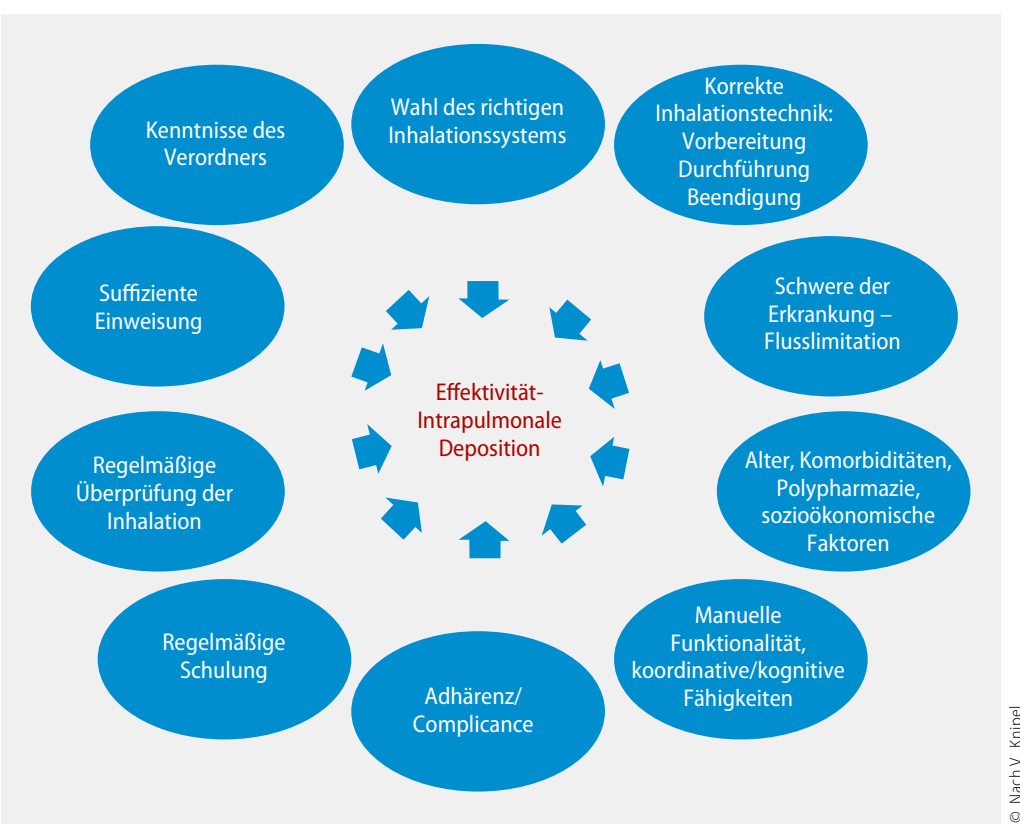

1 Eine effektive Inhalationstherapie mit ausreichender intrapulmonaler Deposition der Wirkstoffe wird von vielen Faktoren beeinflusst.
Die Adhärenz des Patienten hängt auch davon $\mathrm{ab}$, ob er positive Therapieeffekte spürt. Sie liegt bei chronisch Kranken in entwickelten Ländern erfahrungsgemäß bei rund $50 \%$.

\section{Sterblichkeit und Wirtschaftlichkeit}

Eine Subgruppenanalyse der TORCH-Studie (Vestbo J et al., Thorax 2009,64:939-43) hat ergeben, dass sich bei nicht adhärenten COPD-Patienten die Sterblichkeit binnen drei Jahren verdreifacht hat.

Unter Aspekten der Wirtschaftlichkeit sei der Inhalator am teuersten, der nicht richtig benutzt wird, betonte Apothekerin Sabine Müller vom Klinikum Konstanz. A \& O der wirtschaftlichen Verordnung von Atemwegstherapeutika sei daher die intensive Einweisung und Schulung der korrekten Handhabung mit regelmäßiger Überprüfung der Technik. Einen Wechsel des einmal geschulten Inhalationssystems in der Apotheke können Ärzte durch Setzen des Aut-idem-Kreuzes verhindern.

Dagmar Jäger-Becker

\title{
Pfiffige Ideen gesucht - jetzt bewerben!
}

\section{Ab sofort können Mediziner mit guten Ideen wieder gewinnen. Wie's geht, hat 2016 ein Ärztenetz gezeigt.}

Eis und Schneegestöber halten Mediziner des Ärztenetzes Medizin und Mehr (MuM) nicht davon ab, eine Visite bei ihren $\mathrm{Pa}$ tienten im Pflegeheim zu machen. Dafür müssen sich die Ärzte auch nicht in Gefahr begeben, denn sie lassen sich per TabletPC zuschalten. Die Idee der elektronische Visite (elVi $\left.{ }^{\circledast}\right)$ ist für Arzt und Patient so nutzenbringend und innovativ, dass sie mit dem Praxis-Preis 2017 ausgezeichnet wurde.

Das Projekt sorge dafür, dass der Transport zum Arzt nur noch in dringenden Fällen nötig sei, so Dr. Hans-Jürgen Beckmann, Vorstand des MuM-Ärztenetzes beim Empfang des Preises 2016 in Berlin. Auch in diesem Jahr suchen die Fachverlagsgruppe Springer Medizin, zu der auch die „PneumoNews“ gehört, und UCB Innere Medizin Erfolgsrezepte für die Arztpraxis, wie das von Beckmann und seinen Kollegen. Allerdings sind nicht nur weitreichende telemedizinische Ideen gefragt. Auch kleine Einfälle, die dabei helfen, die Versorgung der Patienten zu verbessern - oder den Alltag als Arzt erleichtern - können groß raus kommen.

„Unser Ziel ist es, einen Mehrwert für Patienten zu schaffen. Sie sollen in Arztpraxen adäquat und schnell behandelt werden“, betont Karlheinz Gast, Geschäftsführer von UCB Innere Medizin, mit Blick auf die neue Runde des Ideen-Wettbewerbs, der
2017 zum 7. Mal ausgerichtet wird. Die Themenbandbreite, in der Ärzte mit kreativen Einfällen ihre Arbeit optimieren können, scheint grenzenlos: Bürokratieabbau, Ärztemangel auf dem Land, Qualitätsmanagement, Delegation, Compliance, Marketing, Abrechnung, Mitarbeiterführung und der Kampf gegen Volkskrankheiten wie Diabetes oder COPD sind Bereiche, in denen auch im Kleinen gepunktet wird.

Den besten Ideen winken interessante Preise: Der 1. Preis ist ein dreitägiger Ausbildungslehrgang mit Abschluss ,geprüfte Assistenz für Versorgung und Prävention (Better Care Assistenz)“ von der Unternehmensberatung HCC Better Care aus Köln. Zudem können sich die 10 besten Ideengeber über Fachund Sachliteratur von Springer Medizin und HCC freuen.

Gewinner und Top-Bewerber aus 2016 werden wir noch ausführlicher vorstellen.

Marco Hübner

Weitere Infos und Anmeldung unter aerztezeitung.de/erfolgsrezept

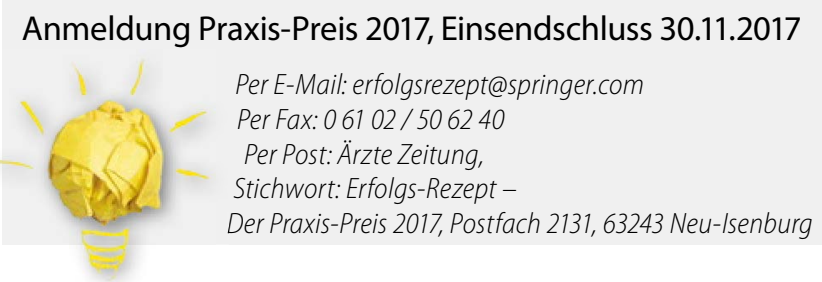

